Research Paper

\title{
Effects of Hypothyroidism on Expression of CRMP2B and ARPC5 during Development of the Rat Frontal Cortex
}

\author{
Chun-rong Liu ${ }^{\bowtie}$, Jun Miao, Yong-liang Zhang, Ya-min Liu, Bao-guo Yu \\ Department of Pathology, Affiliated Hospital of Logistics University of Chinese People's Armed Police Force, Tianjin 300162, China.
}

$\triangle$ Corresponding author: Department of Pathology, Affiliated Hospital of Logistics University of Chinese People's Armed Police Force, ChengLin Road No 220, HeDong District, Tianjin 300162, China. Telephone: +86 022 60578721. E-mail address: lcr68@163.com. (Liu Chun-rong).

(C) Ivyspring International Publisher. This is an open-access article distributed under the terms of the Creative Commons License (http://creativecommons.org/ licenses/by-nc-nd/3.0/). Reproduction is permitted for personal, noncommercial use, provided that the article is in whole, unmodified, and properly cited.

Received: 2012.12.03; Accepted: 2013.01.23; Published: 2013.02.12

\begin{abstract}
Congenital hypothyroidism $(\mathrm{CH})$ can lead to irreversible central nervous system (CNS) damage. However, the pathogenesis of the developmental brain disorders caused by $\mathrm{CH}$ has not been completely elucidated. ARPC5 and CRMP2 are closely associated with neurite outgrowth in brain development. Thus, the aim of the present study was to determine whether CRMP2B and ARPC5 expression is altered in the developing cerebral cortex of rats with $\mathrm{CH}$. Control rats and rats with hypothyroidism were sacrificed at birth and at 15 days postpartum. We performed qRT-PCR to detect differences in the crmp2B and arpc5 mRNA expression in the right half of the frontal cortex of these rats. Western blotting was then used to detect differences in CRMP2B and ARPC5 protein expression. Furthermore, immunohistochemical analysis was performed on the left half of the frontal cortex to detect abnormal localization of CRMP2B and ARPC5. Results showed increased expression of the nuclear short isoform of CRMP2B and decreased expression of full-length CRMP2B and ARPC5 in cortical neurons of rats with hypothyroidism. These findings demonstrate that reduced levels of thyroid hormones can inhibit the expression of full-length CRMP2B and ARPC5 and promote nuclear transformation of the short isoform of CRMP2B. CRMP2B and ARPC5 may participate in CNS injury mediated by hypothyroidism by inducing neurite outgrowth inhibition and cytoskeletal protein disorganization.
\end{abstract}

Key words: CRMP2B; ARPC5; congenital hypothyroidism; frontal cortex; rat.

\section{Introduction}

Congenital hypothyroidism $(\mathrm{CH})$ is a common children endocrine disease. $\mathrm{CH}$ is a condition of thyroid hormone deficiency present at birth. Approximately 1 in 4000 newborn infants has a severe deficiency of thyroid function, while even more have mild or partial degrees. Around the world, the most common cause of congenital hypothyroidism is iodine deficiency [1], but in most of the developed world and areas of adequate environmental iodine, cases are due to a defect of development of the thyroid gland itself.
$\mathrm{CH}$ has a significant long-term impact on the behavior, locomotor ability, speech, hearing and cognition of children [2]. Delay in restoring normal thyroid status in the neonate can lead to irreversible central nerve system damage. Even with prompt thyroid supplementation following the diagnosis of neonatal hypothyroidism, subtle abnormalities of language, visuospatial impairments and lower IQ can still occur later in childhood [3]. To date, the pathogenesis of developmental brain disorders caused by $\mathrm{CH}$ has not 
been completely elucidated.

In a previous study, we used two-dimensional gel electrophoresis to perform an individual comparative proteome analysis of the cerebral cortex from 10 neonatal rats with $\mathrm{CH}$ and 10 healthy neonatal rats [4]. We identified alterations in the expression of seven different proteins, raising the possibility that these proteins may be valuable to clarify brain dysfunction caused by $\mathrm{CH}$. Of these seven proteins, we focused on the actin-related protein 2/3 complex subunit 5 (ARPC5) and collapsin response mediator protein-2B (CRMP2B) because they are closely associated with neurite outgrowth in brain development [5-8]. To date, the spatiotemporal expression and the function of CRMP2B and ARPC5 have not been studied in the developing brain of animal models of hypothyroidism. We therefore sought to investigate whether these proteins might be involved in the pathogenesis of $\mathrm{CH}$.

\section{Materials and methods}

\section{Animals and sampling}

All animals were obtained from the Experimental Animal Centre of the Academy of Military Medical Sciences of China. The study was approved by the Ethics Review Committee of Animal Research of Logistics University of Chinese People's Armed Police Force. Ten pregnant Wistar rats were randomly divided equally into experimental and control groups. Each pregnant rat from the experimental group was intragastrically administered $2.5 \mathrm{ml}$ of $1 \%$ propylthiouracil daily from embryonic day 15 (E15) until postnatal day 15 (P15) [9]. The control group was intragastrically administered $2.5 \mathrm{ml}$ of normal saline daily from E15 until P15. Neonates were weighed prior to sacrifice at birth (P1) and at P15. Blood samples were collected and serum levels of the thyroid hormones, free thyroxine $\left(\mathrm{FT}_{3}\right)$ and free triiodothyronine $\left(\mathrm{FT}_{4}\right)$, and thyroid-stimulating hormone (TSH) were determined by radioimmunoassay (performed by the Radiology Institute of Tianjin Medical University). The right half of the frontal cortex was isolated immediately and frozen in liquid nitrogen. The left half of the frontal cortex was fixed with $4 \%$ formalin and embedded in paraffin. Each subgroup comprised 8 neonatal rats $(n=8)$.

\section{Quantitative reverse-transcription polymerase chain reaction ( $q R T-P C R)$}

Total RNA was isolated from the cerebral cortex of neonate rats with Trizol reagent (Invitrogen) and the DNA was degraded by RNase-Free DNaseI (Fermentas). The RNA was subjected to reverse transcription to synthesize cDNA using the PrimeScript ${ }^{\mathrm{TM}}$
RT reagent (Perfect Real Time) kit (Takara Biotechnology Co., Ltd. Dalian, China). The cDNA samples (100 ng) were prepared for qRT-PCR using a SYBR Premix Ex Taq ${ }^{\mathrm{TM}}$ (Perfect Real Time) kit (Takara Biotechnology Co., Ltd. Dalian, China). The crmp2 primer sequences were 5'-CAC CAA TGG CAC TGA GGA GC-3' (sense) and 5'-CCC ACG GAG ATA CGA CC-3' (antisense), and yielded an amplicon of $150 \mathrm{bp}$. The arpc5 primer sequences were 5'-AAG TTC GTG GAC GAG GAG G-3' (sense) and 5'-GTA GGG CAG CGG TCA TGT TTC-3' (antisense), resulting in a product of $100 \mathrm{bp}$. The ubiquitin C (internal control) primer sequences were $5^{\prime}$ - TCG TAC CTT TCT CAC CAC AGT ATC TAG -3' (sense) and 5'- GAA AAC TAA GAC ACC TCC CCA TCA -3' (antisense), and yielded an amplicon of $82 \mathrm{bp}$. The qRT-PCR amplifications were carried out using a Roche LightCycler. All reactions were performed in triplicate and included negative control reactions lacking cDNA.

\section{Western blotting}

Samples of rat cerebral cortex $(100 \mathrm{mg})$ were homogenized on ice in $1 \mathrm{ml}$ of lysis buffer $(7 \mathrm{M}$ Carbamide, $2 \mathrm{M}$ thiourea, $20 \mathrm{mM}$ Tris- $\mathrm{HCl}$, 4\% CHAPS, $60 \mathrm{mM}$ DTT and 1\% cocktail protease inhibitors). The homogenates were frozen and thawed three times, underwent shaking for $1 \mathrm{~h}$, and were then centrifuged at $14,000 \times \mathrm{g}$ for $30 \mathrm{~min}$ at $4^{\circ} \mathrm{C}$. An equal volume of supernatant from ten rats from the same group were combined and centrifuged at 26,000 $\times \mathrm{g}$ for $1 \mathrm{~h}$ at $4^{\circ} \mathrm{C}$. Proteins were extracted from the supernatant and quantified using a BCA Protein Assay kit (Beijing Biomed Co., Ltd., China). Proteins $(100 \mu \mathrm{g})$ were run on a precast $10 \%$ polyacrylamide gel and transferred to a PVDF membrane (Millipore) at $15 \mathrm{~V}$ for $25 \mathrm{~min}$ using a Trans-Blot SD Transfer Cell (Bio-Rad). Non-specific staining was blocked by incubating the membranes in 5\% nonfat milk in TBST for $2 \mathrm{~h}$ while rotating at room temperature. Membranes were then incubated sequentially with primary (overnight at 4 ${ }^{\circ} \mathrm{C}$ ) and secondary antibodies ( $2 \mathrm{~h}$ at room temperature). The following primary antibodies were used: anti-CRMP2B (1:500 dilution; kindly provided by Dr Miao Jun from the Institute of Orthopaedics, Tianjin Hospital, Tianjin, China. The peptide sequence used to generate specific antisera was ${ }^{454}$ LEDGTLHVTEGS 465 , targeting the CRMP2B C-terminal region); anti-ARPC2 (1:2,000; Abcam); and anti-GAPDH (1:5,000; Abmart). The appropriate secondary antibodies (Abmart) were used at a dilution of 1:5,000. Target proteins were visualized on the membranes using enhanced chemiluminescence (Immobilon ${ }^{\mathrm{TM}}$ Western Chemiluminescent HRP Substrate, Millipore Corporation, USA) and photographed using a Kodak Medical X-ray processor. Data were acquired 
with SynGene 3.07.03 analysis software.

\section{Immunohistochemistry}

Immunohistochemical detection of CRMP2B and ARPC5 was performed with formalin-fixed paraffin-embedded frontal cortex specimens. Sections (5 $\mu \mathrm{m})$ were deparaffinized with xylene and rehydrated with graded alcohol solutions. Endogenous peroxidase activity was quenched by boiling the sections in $10 \mathrm{mM}$ citrate buffer ( $\mathrm{pH}$ 6.0) for $3 \mathrm{~min}$, followed by cooling at room temperature for more than $20 \mathrm{~min}$. Sections were incubated with anti-CRMP2B antibody (1:200), or anti-ARPC5 antibody (1:200), overnight at $4^{\circ} \mathrm{C}$. Sections were then incubated with the appropriate horseradish peroxidase (HRP)-conjugated secondary antibody (PV6001, Zhongshan Goldenbridge Biotechnology Co, Ltd, Beijing, China) for $30 \mathrm{~min}$ at $37^{\circ} \mathrm{C}$ and the color was developed with 3, 3'-diaminobenzidine for 1 to $2 \mathrm{~min}$. The negative control involved the omission of primary antibody. All slides were analyzed by two independent observers.

\section{Evaluation of immunohistochemical staining}

Cortical layer III and IV with high numbers of CRMP2B- or ARPC5-positive cells were identified at low magnification $(\times 10$ ocular and $\times 10$ objective) as "hot spots". We selected ten hot spots per section to observe at higher magnification $(\times 10$ ocular and $\times 40$ objective, high power field). Using a grid in the ocular lens (Olympus 100x), and counted all neurons and positively stained cells in every high power field. The mean percentage of positively stained cells was used to evaluate the expression of the protein in a section. The fraction of CRMP2B- or ARPC5-positive cells was scored as $0(0 \%$ positive cells), $1(1 \%-25 \%$ positive cells), 2 ( $26 \%-50 \%$ positive cells), 3 ( $50 \%-75 \%$ positive cells) or 4 ( $>75 \%$ positive cells). The intensity of CRMP2B or ARPC5 immunostaining was scored as 0 (negative), 1 (weak), 2 (intermediate) or 3 (strong). The intensity score (0-3) was multiplied by the fraction score (0-4) and a final score was assigned 0 (negative, -), 1-4 (weak expression, +), 5-8 (moderate expression, ++), and 9-12 (strong expression, +++). Dendritic elongation was measured by ocular micrometer.

\section{Statistical analysis}

Fluorescent data obtained from qRT-PCR were processed using the LinRegPCR method to obtain the relative quantity of target mRNA [10-12]. Differences in CRMP2 and ARPC5 gene and protein expression between groups were assessed by analysis of variance (ANOVA). To assess CRMP2 and ARPC5 staining intensity, we used the nonparametric Mann-Whitney test for the comparison of two groups. Statistical analyses were performed using SPSS version 13.0. In all cases, $p<0.05$ was considered significant.

\section{Results}

\section{Phenotype of rats with hypothyroidism}

The mortality rate of rats with hypothyroidism (50\%) was higher than that of the control group. Rats with hypothyroidism grew slowly, were small and had short tails. They opened their eyes late; their hair grew slowly and was sparse, and they were weaned late. Their balance was poor and they reacted slowly to stimuli. The body weight of rats with hypothyroidism was lower than that of control rats at both P1 and P15 $\left(t_{\mathrm{P} 1}=-8.074, t_{\mathrm{P} 15}=-7.891, P<0.01\right)$. Serum FT 3 and $\mathrm{FT}_{4}$ levels were also lower in rats with hypothyroidism compared with control rats at both P1 and $\mathrm{P} 15\left(t_{\mathrm{P} 1}=-5.386, t_{\mathrm{P} 15}=-6.624, P<0.01\right.$ and $t_{\mathrm{P} 1}=-7.618$, $t_{P 15}=-12.806, P<0.01$, respectively). The level of serum TSH rats at P1 and P15 with hypothyroidism was higher than that of control rats at the same stage $\left(t_{\mathrm{P} 1}=\right.$ -9.789, $\left.t_{\mathrm{P} 15}=-11.448, p<0.01\right)$ (Table 1).

\section{Differential expression of crmp2 and arpc5 mRNA in the frontal cortex of rats with hy- pothyroidism compared with healthy rats}

There was no statistically significant difference in crmp 2 and arpc5 mRNA expression levels between rats with hypothyroidism and control rats at $\mathrm{P} 1\left(P_{\text {crmp2 }}\right.$ $\left.=0.96, P_{\text {arpc }}=0.268\right)$. Both $c r m p 2$ and arpc5 mRNA expression levels were upregulated in the frontal cortex of control rats and rats with hypothyroidism at P15 $\left(P_{\text {crmp2 }}<0.001, P_{\text {arpc } 5}<0.001\right)$. The mRNA expression levels of crmp2 and $\operatorname{arpc5}$ were lower in the frontal cortex of hypothyroidism rats compared with healthy rats at $\mathrm{P} 15\left(P_{\text {crmp } 2}=0.008, P_{\text {arpc } 5}<0.001\right)$ (Fig. $1 \mathrm{a}$ and $1 b)$.

Table I. Body weight (BW) and serum $\mathrm{FT}_{3}, \mathrm{FT}_{4}$ and $\mathrm{TSH}$ levels in each group $(n=8$, mean \pm standard deviation).

\begin{tabular}{llllll}
\hline Group & & $\mathrm{BW}(\mathrm{g})$ & $\mathrm{FT}_{3}(\mathrm{pmol} / \mathrm{L})$ & $\mathrm{FT}_{4}(\mathrm{pmol} / \mathrm{L})$ & $\mathrm{TSH}(\mathrm{IU} / \mathrm{mL})$ \\
\hline Control & $\mathrm{P} 1$ & $5.54 \pm 0.14$ & $0.26 \pm 0.05$ & $4.82 \pm 1.10$ & $0.48 \pm 0.10$ \\
& $\mathrm{P} 15$ & $24.66 \pm 2.68$ & $1.40 \pm 0.31$ & $88.31 \pm 20.10$ & $0.70 \pm 0.12$ \\
Hypothyroid & P1 & $4.82 \pm 0.24^{*}$ & $0.15 \pm 0.04^{*}$ & $2.03 \pm 0.37^{*}$ & $0.90 \pm 0.09^{*}$ \\
& $\mathrm{P} 15$ & $14.33 \pm 3.15^{*}$ & $0.63 \pm 0.20^{*}$ & $6.53 \pm 2.03^{*}$ & $1.27 \pm 0.11^{*}$ \\
\hline
\end{tabular}

${ }^{*} \boldsymbol{P}<0.01$ vs control group. 


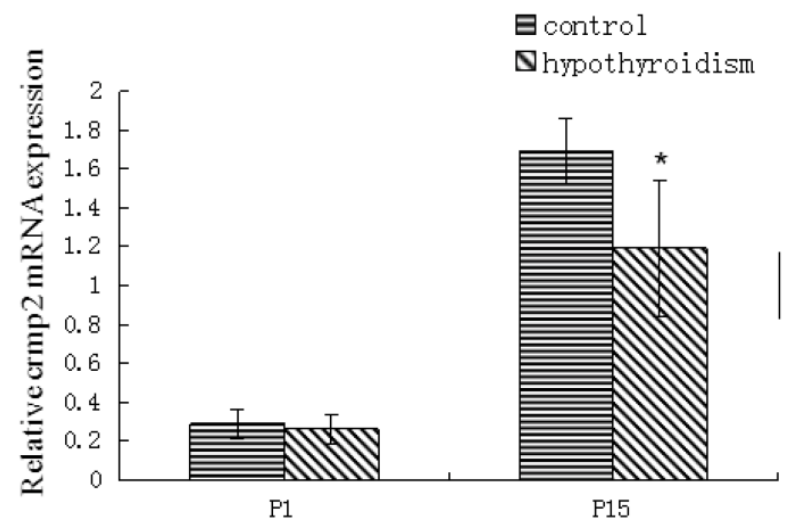

a

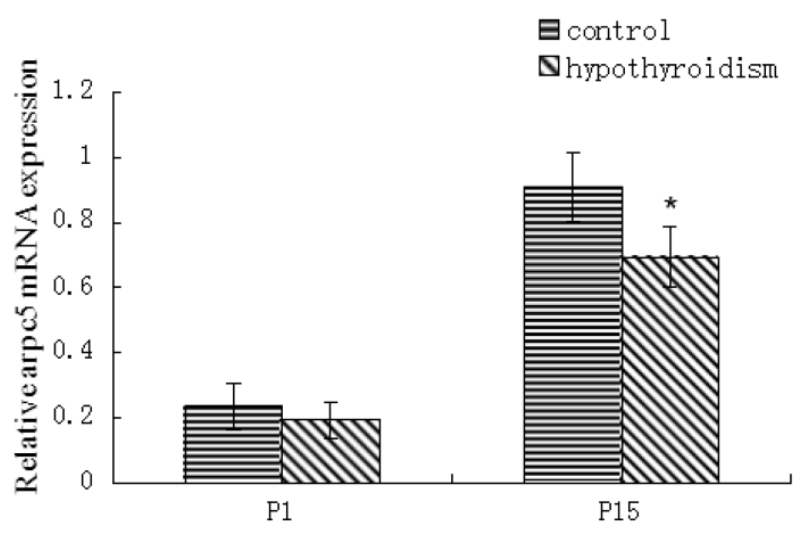

b

Fig. I. (a) Differential expression of $c r m p 2$ mRNA in the frontal cortex of normal rats and rats with hypothyroidism. (b) Differential expression of arpc5 mRNA in the frontal cortex of normal rats and rats with hypothyroidism. $* P<0.05$ compared with control.

\section{Differential expression of CRMP2 and ARPC5 protein in the frontal cortex of rats with hy- pothyroidism compared with healthy rats}

No statistically significant difference between the protein expression level of full-length CRMP2B $(64 \mathrm{kDa})$ was observed between rats with hypothyroidism and normal rats at $\mathrm{P} 1(P=0.319)$. In control rats, full-length CRMP2B protein expression was higher at P15 than at P1 $(P<0.001)$. In rats with hypothyroidism, there was no difference between full-length CRMP2B expression at P1 and P15 $(P=$ 0.342). Full-length CRMP2B protein expression was higher in the frontal cortex of control rats than in hypothyroidism rats at $\mathrm{P} 15(P=0.004)$ (Fig. $2 \mathrm{a}$ and $2 \mathrm{c}-\mathrm{i})$.

CRMP2B (62 kDa) was not detected in normal rats and hypothyroidism rats at P1. No statistically significant difference between the protein expression level of CRMP2B (62 kDa) was observed between rats with hypothyroidism and normal rats at P15 $(P=$ 0.733).

No difference in the level of expression of the short CRMP2B (58 kDa) isoform was detected at P1 in the frontal cortex of hypothyroidism rats compared with control rats $(P=0.319)$. Short CRMP2B expression was lower in the frontal cortex of control rats than in hypothyroidism rats at P15 $(P<0.001)$. The expression of short CRMP2B was upregulated at P15 compared with P1 rats with hypothyroidism $(P<$ $0.001)$, but was downregulated at P15 compared with P1 control rats $(P=0.017)$ (Fig. 2a and 2c-ii).

There was no statistically significant difference between the level of expression of ARPC5 in the frontal cortex of hypothyroidism rats compared with healthy rats at $\mathrm{P} 1(P=0.538)$. In both groups of rats, ARPC5 protein expression was higher at P15 than at
P1 $\left(P_{\text {control }}<0.001, P_{\mathrm{CH}}<0.001\right)$. The expression of ARPC5 was decreased in the frontal cortex of hypothyroidism rats compared with control rats at P15 $(P<$ 0.001) (Fig. 2b and 2c-iii).

\section{Abnormal localization of CRMP2 and ARPC5 in the frontal cortex of rats with hypothyroid- ism compared with control rats}

CRMP2B staining was scattered throughout the frontal cortex of P1 rats. CRMP2B was predominantly distributed in neurites and the cytoplasm of neurons. No difference was observed between the intensity of CRMP2B staining in control rats and rats with hypothyroidism at this stage $(Z=-0.968, P=0.333)$ (Table 2 ). CRMP2B was expressed diffusely throughout the six layers of frontal cortex of P15 rats, and was expressed in the nucleus and cytoplasm of neurons, as well as in neurites. Cortical layer III and IV were emphatically studied. The intensity of CRMP2B staining was stronger in control rats than in rats with hypothyroidism at P15 $(Z=-2.031, P=0.042)$ (Table $3)$. However, the number of CRMP2B-positive nuclei was greater in rats with hypothyroidism compared with control rats at this stage $(t=19.359, P<0.001)$ (Table 6). Diminished dendritic branching and elongation was observed in neurons with intense nuclear CRMP2B staining $(t=-7.364, P<0.001)$ (Fig.3).

ARPC5 was expressed diffusely throughout the six layers of the frontal cortex and was predominantly found in neurites. Cortical layer III and IV were emphatically studied. No difference was found in the intensity of ARPC5 staining in rats with hypothyroidism compared with control rats at $\mathrm{P} 1(Z=-0.522$, $P=0.602$ ) (Table 4$)$. The intensity of ARPC5 staining was lower in rats with hypothyroidism than in control rats at P15 $(Z=-2.450, P=0.014)$ (Table 5 and Fig. 4). 

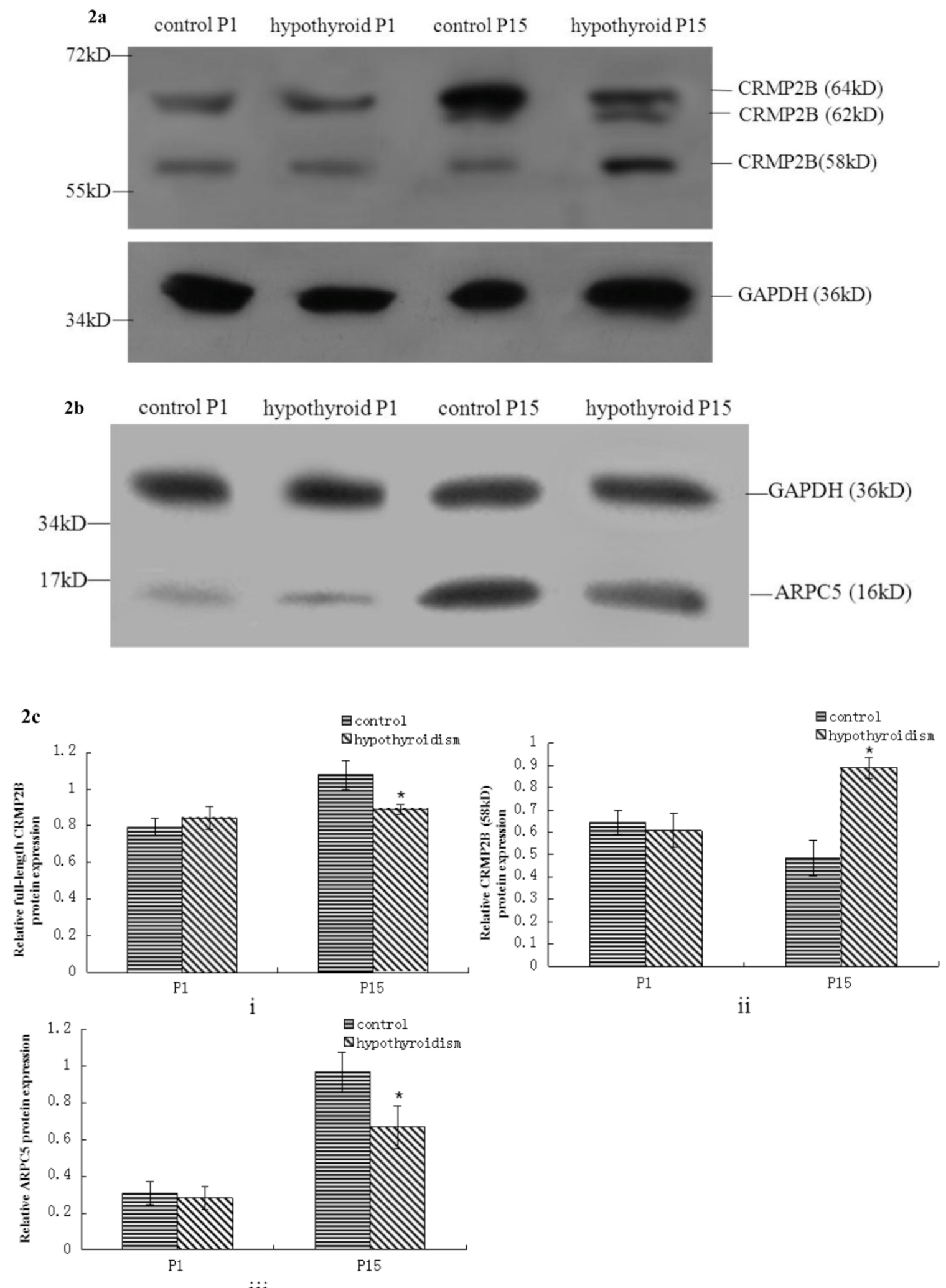

iii

Fig. 2. Western blotting results: (a) Differential expression of CRMP2B protein in the frontal cortex of normal rats and rats with hypothyroidism. (b) Differential expression of ARPC5 protein in the frontal cortex of normal rats and rats with hypothyroidism. (c) The quantification of the bands shown in Fig2a and Fig2b. i: quantification of full-length CRMP2B $(64 \mathrm{kDa})$ protein expression; ii: quantification of CRMP2B (58 kDa) protein expression; iii: quantification of ARPC5 protein expression. $* P<0.05$ compared with control. 
Table 2. Variations in CRMP2B expression at PI in the cortical layer III and IV of control rats and hypothyroidism rats.

\begin{tabular}{|c|c|c|c|c|c|c|}
\hline \multirow[t]{2}{*}{ Group } & \multirow[t]{2}{*}{$\mathrm{n}$} & \multicolumn{3}{|c|}{ Intensity of staining } & \multirow[t]{2}{*}{ Z } & \multirow[t]{2}{*}{$\mathrm{P}$} \\
\hline & & + & ++ & +++ & & \\
\hline Control & 8 & 3 & 5 & 0 & -0.968 & 0.333 \\
\hline Hypothyroid & 8 & 5 & 3 & 0 & & \\
\hline
\end{tabular}

Table 3. Variations in CRMP2B expression at PI5 in the cortical layer III and IV of control rats and hypothyroidism rats.

\begin{tabular}{|c|c|c|c|c|c|c|}
\hline \multirow[t]{2}{*}{ Group } & \multirow[t]{2}{*}{$\mathrm{n}$} & \multicolumn{3}{|c|}{ Intensity of staining } & \multirow[t]{2}{*}{$\mathrm{Z}$} & \multirow[t]{2}{*}{$\mathrm{P}$} \\
\hline & & + & ++ & +++ & & \\
\hline Control & 8 & 0 & 1 & 7 & -2.031 & 0.042 \\
\hline Hypothyroid & & 1 & 4 & 3 & & \\
\hline
\end{tabular}

Table 4. Variations in ARPC5 expression at PI in the cortical layer III and IV of control rats and hypothyroidism rats.

\begin{tabular}{|c|c|c|c|c|c|c|}
\hline \multirow{2}{*}{ Group } & \multirow[t]{2}{*}{$\mathrm{n}$} & \multicolumn{3}{|c|}{ Intensity of staining } & \multirow[t]{2}{*}{ Z } & \multirow[t]{2}{*}{$\mathrm{P}$} \\
\hline & & + & ++ & +++ & & \\
\hline Control & 8 & 2 & 6 & 0 & -0.522 & 0.602 \\
\hline Hypothyroid & & 3 & 5 & 0 & & \\
\hline
\end{tabular}

Table 5. Variations in ARPC5 expression at PI5 in the cortical layer III and IV of control rats and hypothyroidism rats.

\begin{tabular}{|c|c|c|c|c|c|c|}
\hline \multirow[t]{2}{*}{ Group } & \multirow[t]{2}{*}{$\mathrm{n}$} & \multicolumn{3}{|c|}{ Intensity of staining } & \multirow[t]{2}{*}{ Z } & \multirow[t]{2}{*}{$\mathrm{P}$} \\
\hline & & + & ++ & +++ & & \\
\hline Control & 8 & 0 & 1 & 7 & -2.450 & 0.014 \\
\hline Hypothyroid & 8 & 1 & 5 & 2 & & \\
\hline
\end{tabular}

Table 6. Comparison of the number of CRMP2B-positive nuclei at PI5 in the cortical layer III and IV of control rats and hypothyroidism rats (mean \pm standard deviation).

\begin{tabular}{lrlll}
\hline Group & $n$ & Number & $t$ & $P$ \\
\hline Control & 8 & $11.925 \pm 3.547$ & 19.359 & $<0.001$ \\
Hypothyroid & 8 & $29.050 \pm 4.326$ & & \\
\hline
\end{tabular}
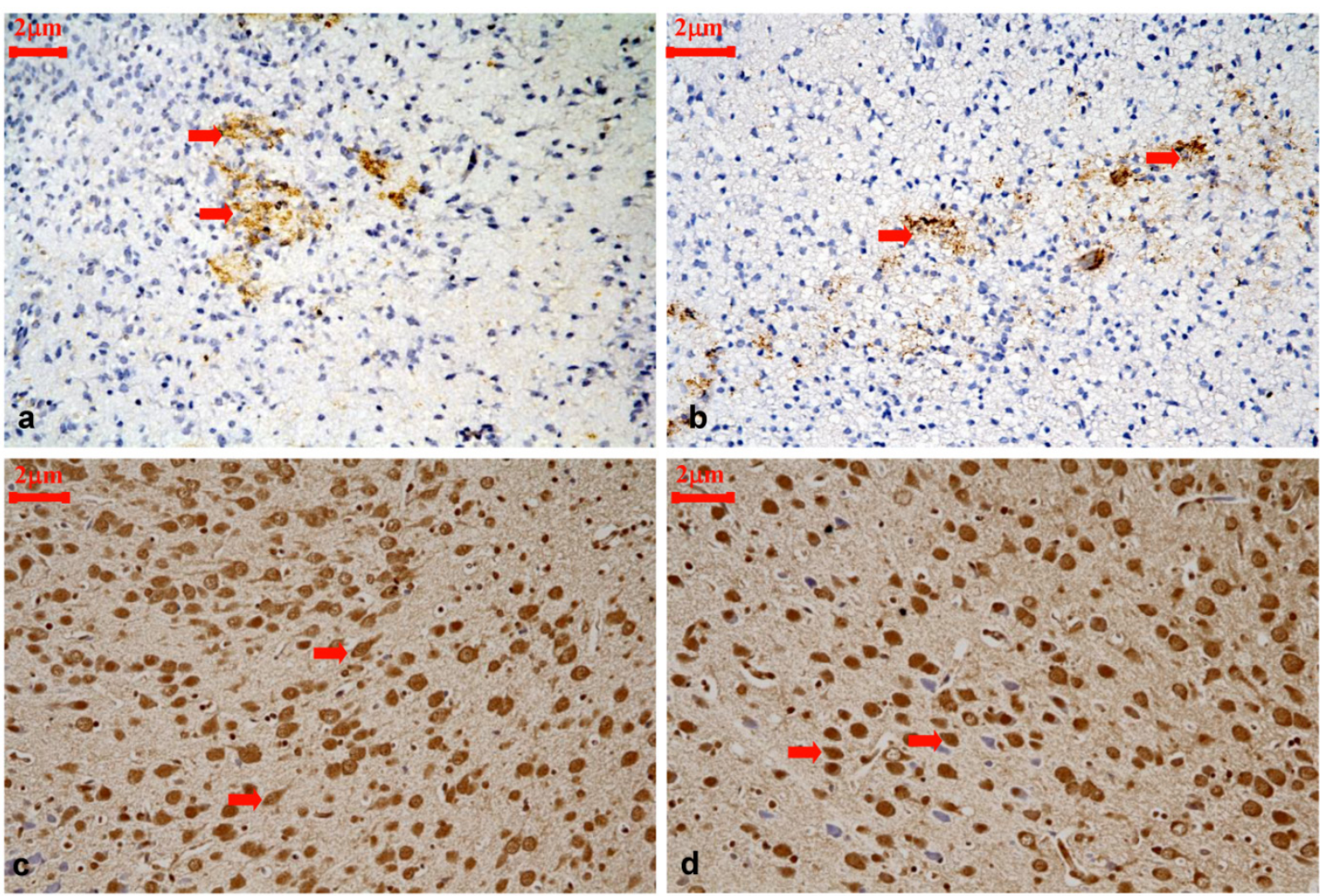

Fig. 3. Representative immunohistochemical staining for CRMP2B protein expression in the cortical layer III and IV of control rats and hypothyroidism rats during development, 400×. (a): PI control, CRMP2B staining was scattered and predominantly distributed in neurites and the cytoplasm of neurons $(\Rightarrow$ red). (b): PI hypothyroidism, the distribution and cellular location were similar to PI control group. (c): PI5 control, majority of CRMP2B staining cells are cytoplasmic staining cells with longer neurite $(\Rightarrow$ red). (d): PI5 hypothyroidism. majority of CRMP2B staining cells are nuclear staining cells with shorter neurite $(\Rightarrow$ red). 

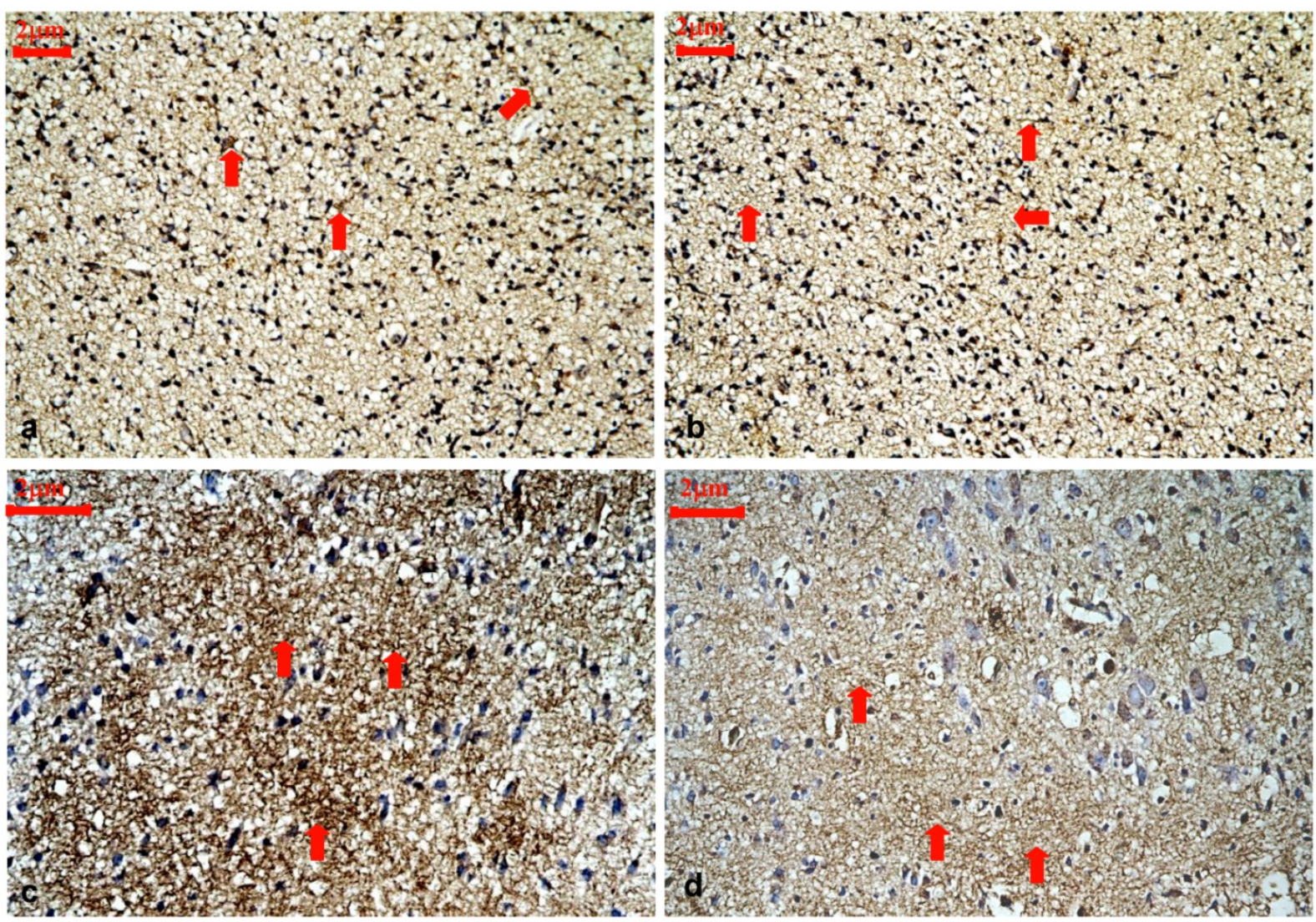

Fig. 4. Representative immunohistochemical staining for ARPC5 protein expression in the cortical layer III and IV of control rats and hypothyroidism rats during development, 400x. (a): PI control. ARPC5 was diffusely expressed and predominantly found in neurites $(\Rightarrow$ red). (b):PI hypothyroidism. The distribution and cellular location were similar to PI control group. (c): PI5 control. ARPC5 was found in neurites. The intensity of ARPC5 staining was higher in control rats $(\Rightarrow$ red). (d): PI5 hypothyroidism. ARPC5 was found in neurites. The intensity of ARPC5 staining was lower in hypothyroid rats $(\Rightarrow$ red).

\section{Discussion}

Investigators frequently make reference to a "critical period" in which appropriate thyroid hormone levels are essential for normal brain development. In humans, this period is generally considered to begin late in gestation and to extend through 1-2 years of age. The equivalent period in rats is thought to occur in the first 10-12 days postpartum [10]. Proliferation of axons and dendrites, synapse formation, gliogenesis, and myelination occurs during this critical period in the cerebrum [11]. Because CRMP2 and ARPC5 are involved in neuronal outgrowth and synapse formation [5-8], we chose to study CRMP2 and ARPC5 at the two key time points at which these processes occur: at birth (P1) and 15 days postpartum (P15). The phenotypic features of rats exposed to propylthiouracil in utero was consistent with those observed in congenital hypothyroidism, thus validating this animal model of hypothyroidism.

CRMP2 was originally identified as a signaling molecule required for growth cone collapse of dorsal root ganglion neurons in response to semaphorin-3A
[12]. The overexpression of CRMP2 in neurons induces the formation of multiple axons, and the expression of a dominant-negative form of CRMP2 or the knockdown of CRMP2 suppresses axon formation [13]. Together, these findings suggest that CRMP2 has a positive effect on axonal extension and plays a crucial role in axon-dendrite specification and axon regeneration. Various isoforms of CRMP2 have been identified, including CRMP2A and CRMP2B. There are also three isoforms of CRMP2B which differ in their molecular weight $(58,62$ and $64 \mathrm{kDa})$ [14, 15]. Full-length CRMP2B (64 kDa) is restricted to the cytoplasm of neurons, where it co-localizes with actin. The co-localization of these two proteins suggests that CRMP2B might mediate actin reorganization through either direct or indirect interaction. In addition, full-length CRMP2B can regulate axonal growth in cortical neurons. The length of primary axons in neurons expressing full-length CRMP2B was longer (1.6-fold) than that of nontransfected neuronal cells, which expressed endogenous CRMP2B. CRMP2B (62 $\mathrm{kDa}$ ) is located in the cytoplasm of neurons. Chung et al reported the expression level of CRMP2B (62 kDa) 
was decreased with the brain ischemic duration increased. CRMP2B (62 kDa) may be an important candidate controlling ischemic stroke [16]. In the present study, there was no statistically significant difference in CRMP2B (62 kDa) protein expression level between rats with hypothyroidism and control rats, thus we did not attach more attention to it. Short CRMP2B (58 $\mathrm{kDa}$ ) is expressed in both the nucleus and cytoplasm of neurons, but does not co-localize with actin [17]. Thus, short CRMP2B is unlikely to play a role in actin reorganization. However, the function of short nuclear CRMP2B has not been extensively studied.

To be imported into the nucleus, proteins need to be actively transported through the nuclear pore by importins. This process is dependent on the interaction of importins with a nuclear localization signal (NLS) in the protein being transported [18]. The NLS motif in CRMP2B comprises residues Arg ${ }^{471}$ Lys $^{472}$. In full-length CRMP2B, an intrinsically unstructured and bulky C-terminal region (residues 489-572) is located near the NLS motif. Thus, in the full-length protein, the NLS motif might be buried within the bulky C-terminal region and be inaccessible for nuclear targeting. However, the short CRMP2B isoform is devoid of a part of this unstructured C-terminal region and, thus, the NLS motif may be exposed, explaining why only the short CRMP2B isoform is located in the nucleus. The molecules and mechanisms that promote or inhibit translocation of the short CRMP2B isoform into the nucleus have not yet been identified.

In the present study, we showed that the expression of the full-length and short isoforms of CRMP2B is developmentally regulated in the rat frontal cortex. A particularly intriguing finding of this study is that both mRNA and protein levels of the full-length isoform of CRMP2B are downregulated in the developing frontal cortex of rats with hypothyroidism compared with healthy rats. In contrast, the expression of the short isoform of CRMP2B is upregulated in the developing cerebral cortex of rats with hypothyroidism compared with normal rats. Zhang et al confirmed that the decrease of intact CRMP-2 occurs with a concurrent increase of the short CRMP-2 fragment due to calpain-2 proteolysis, in vitro and in vivo, and that the calpain-mediated CRMP-2 proteolysis following traumatic brain injury (TBI) may be associated with neuronal cell injury and neurite damage [19].We also found that dendritic branching and elongation was reduced in neurons with intense nuclear CRMP2B staining (that is, in neurons with increased short CRMP2B). The latter result is in accord with previous reports that full-length CRMP2B induces neurite outgrowth and the appearance of polygonal cells, while short CRMP2B constructs inhibit neurite elongation and produce round cells [17]. Furthermore, it is well established that short CRMP2B inhibits neurite formation and axonal growth and that this inhibition is dependent on the nuclear localization of short CRMP2B. Therefore, we postulate that a proteolytic process might modify CRMP2B following irreversible central nervous system (CNS) injuries caused by hypothyroidism. Such a process could account for the increase we observed in short CRMP2B in rats with hypothyroidism, and higher levels of short CRMP2B may in turn have been responsible for the inhibition of dendritic branching and elongation observed in the same rats.

Taken together, our data indicate that, during the "critical period" of normal brain development, the full length isoform of CRMP2B is dominantly expressed and the nuclear short isoform of CRMP2B is gradually down-regulated. The full length isoform of CRMP2B exerts positive effects on neurite formation and axonal extension. In contrast, the dominant expression of the nuclear short isoform of CRMP2B during brain development of animals with hypothyroidism is likely to have a negative effect on neurite formation and elongation. Our results also indicate that reduced levels of thyroid hormones can inhibit the expression of full-length CRMP2B and promote the nuclear translocation of the short isoform of CRMP2B. Thus, the abnormalities observed in the behavior, locomotor ability, speech, hearing and cognition of children with $\mathrm{CH}$ may be closely associated with reduced neuronal outgrowth and abnormal synapse formation in the brain.

In response to extracellular signals, the Arp2/3 complex nucleates actin filaments to regulate their assembly. The Arp2/3 complex consists of Arp2, Arp3, ARPC1, ARPC2, ARPC3, ARPC4, and ARPC5. Arp2 and Arp3 are believed to form the actin nucleation site. ARPC2 and 4 form the structural core of the complex and also bind to pre-existing actin filaments, while ARPC1, 3, and 5 are involved in coupling activator binding to complex activation [20]. In the crystal structure of the Arp2/3 complex, ARPC5 protrudes from the surface of the complex and interacts with Arp2, ARPC1, and ARPC4 [21]. No specific function has been ascribed to the smallest member of the complex, ARPC5. ARPC5 might form part of an interaction surface that spans several subunits, and the Arp2/3 complex, together with actin filaments, might be altered by ARPC5. The Arp2/3 complex is essential for formation of invadopodia and lamellipodia [22, 23]. Here, we demonstrated that the spatiotemporal expression of ARPC5 is developmentally regulated. The expression of ARPC5 was lower in the frontal 
cortex of P15 rats with hypothyroidism, suggesting that ARPC5 may be a target gene of thyroid hormones. The lower ARPC5 expression at P15 may contribute to deformation of growth cone filopodia and lamellipodia by interfering with actin activation and reorganization.

Axon elongation is a fundamental step for formation of complex neuronal network. Growth cones guide axons to their targets in response to guidance cues, and are located at the tip of the process and consist of filopodia and lamellipodia. The actin cytoskeleton is crucial for determining the shape of growth cone filopodia and lamellipodia. Growth cone collapse is a very important process in the directional elongation of axons and is associated with a net loss of actin filaments at the leading edge of growth cones. CRMP2B mediates axonal guidance by collapsing growth cones during development and this activity is correlated with the reorganization of cytoskeletal proteins [24]. ARPC5 provides an interaction surface for reorganization of cytoskeletal proteins. Thus, in rats with hypothyroidism, the reduced expression of APRC5 and full-length CRMP2B in neurites, together with the increased expression of short CRMP2B in the nucleus, may have contributed to dysfunction of the growth cone by inducing disorganization of cytoskeletal proteins.

In conclusion, to our knowledge, the present study is the first to demonstrate increased expression of the nuclear short isoform of CRMP2B and decreased expression of the full-length isoforms of CRMP2B and ARPC5 in cortical neurons of rats with hypothyroidism. The changes in the levels of these proteins likely induced inhibition of neurite outgrowth, growth cone dysfunction, and cytoskeletal protein disorganization. Our studies provide new insights into the pathogenesis of brain injury caused by hypothyroidism. Considerably more work needs to be done, including investigating the association between CRMP2B and ARPC5, and exploring the mechanism by which reduced serum levels of thyroid hormones induce translocation of short CRMP2 into the nucleus of neurons. This study was an important first step in determining the role of CRMP2B and ARPC5 in mediating the CNS injury caused by hypothyroidism.

\section{Highlights}

1. Reduced levels of thyroid hormones promote the nuclear translocation of short CRMP2B.

2. Reduced levels of thyroid hormones inhibit the expression of full-length CRMP2B.

3. Reduced levels of thyroid hormones inhibit the expression of ARPC5.

\section{Abbreviations}

Arp2/3: actin related protein 2/3 complex; ARPC5: actin-related protein 2/3 complex subunit 5; $\mathrm{CH}$ : congenital hypothyroidism; CNS: central nervous system; CRMP2: collapsin response mediator protein-2; $\mathrm{FT}_{3}$ : free thyroxine; $\mathrm{FT}_{4}$ : free triiodothyronine; HRP: horseradish peroxidase; qRT-PCR: quantitative reverse-transcription polymerase chain reaction; TSH: thyroid-stimulating hormone; P1: postnatal 1d; P15: postnatal 15d.

\section{Acknowledgement}

This research was supported by the National Natural Science Foundation (30471934) and Chinese People's Armed Police Force Foundation (WY200803).

\section{Competing Interests}

The authors have declared that no competing interest exists.

\section{References}

1. Chan S \& Kilby MD. Thyroid hormone and central nervous system development. J Endocrinol. 2000; 165: 1-8.

2. Legrand J. Thyroid hormone effects on growth and development. In: Thyroid Hormone Metabolism. Ed. G Hemmeman. New York:M. Dekker, Inc; 1984: 503-34.

3. Heyerdahl S, Kase BF, Lie SO, et al. Intellectual development in children with congenital hypothyroidism in relation to recommended thyroxine treatment. J Pediatr 1991; 118: 850-7.

4. Liu CR, Yu BG, Liu YQ, et al. Proteomic changes in cerebral cortex of neonatal rats with experimental congenital hypothyroidism. Zhonghua Er Ke Za Zhi. 2011; 49: 209-13.

5. Zhou FQ \& Cohan CS. How actin filaments and microtubules steer growth cones to their targets. J Neurobiol. 2004; 58: 84-91.

6. Boczkowska M, Rebowski G, Petoukhov MV, et al. X-ray scattering study of activated Arp2/3 complex with bound actin-WCA. Structure. 2008; 16: 695-704.

7. Inagaki N, Chihara $\mathrm{K}$, Arimura $\mathrm{N}$, et al. CRMP-2 induces axons in cultured hippocampal neurons. Nat Neurosci. 2001; 4: 781-2.

8. Buck KB, Zheng JQ. Growth cone turning induced by direct local modification of microtubule dynamics. J Neurosci. 2002; 22: 9358-67.

9. Liu CR, Li LY, Shi F, et al. Effects of hyper- and hypothyroid on expression of thyroid hormone receptor mRNA in rat myocardium. J Endocrinol. 2007; 195: 429-38.

10. Porterfield SP, Hendrich CE. The role of thyroid hormones in prenatal and neonatal neurological development--current perspectives. Endocr Rev. 1993; 14: 94-106.

11. Babu S, Sinha RA, Mohan V, et al. Effect of hypothyroxinemia on thyroid hormone responsiveness and action during rat postnatal neocortical development. Exp Neurol. 2011; 228: 91-8.

12. Uchida $\mathrm{Y}$, Ohshima $\mathrm{T}$, Yamashita $\mathrm{N}$, et al. Semaphorin $3 \mathrm{~A}$ signaling mediated by Fyn-dependent tyrosine phosphorylation of collapsin response mediator protein 2 at tyrosine 32. J Biol Chem. 2009; 284: 27393-401.

13. Suzuki Y, Nakagomi S, Namikawa K, et al. Collapsin response mediator protein-2 accelerates axon regeneration of nerve-injured motor neurons of rat. J Neurochem. 2003; 86: 1042-50.

14. Bretin S, Reibel S, Charrier E, et al. Differential expression of CRMP1, CRMP2A, CRMP2B, and CRMP5 in axons or dendrites of distinct neurons in the mouse brain. J Comp Neurol. 2005; 486: 1-17.

15. Kobeissy FH, Ottens AK, Zhang Z, et al. Novel differential neuroproteomics analysis of traumatic brain injury in rats. Mol Cell Proteomics. 2006; 5: 1887-98.

16. Chung MA, Lee JE, Lee JY, et al. Alteration of collapsin response mediator protein-2 expression in focal ischemic rat brain. Neuroreport. 2005; 16:1647-53. 
17. Rogemond V, Auger C, Giraudon P, et al. Processing and Nuclear Localization of CRMP2 during Brain Development Induce Neurite Outgrowth Inhibition. The Journal of Biological Chemistry. 2008; 283: 14751-61.

18. Weis K. Nucleocytoplasmic transport: cargo trafficking across the border. Curr Opin Cell Biol. 2002; 14: 328-35.

19. Zhang Z, Ottens AK, Sadasivan S, et al. Calpain-mediated collapsin response mediator protein-1, -2 , and -4 proteolysis after neurotoxic and traumatic brain injury. J Neurotrauma. 2007; 24: 460-72.

20. Millard TH, Behrendt B, Launay $S$, et al. Identification and Characterisation of a Novel Human Isoform of Arp2/3 Complex Subunit p16-ARC/ARPC5. Cell Motil Cytoskeleton. 2003; 54: 81-90.

21. Robinson RC, Turbedsky K, Kaiser DA, et al. Crystal structure of Arp2/3 complex. Science. 2001; 294: 1679-84.

22. Kinoshita $\mathrm{T}$, Nohata N, Watanabe-Takano $\mathrm{H}$, et al. Actin-related protein $2 / 3$ complex subunit 5 (ARPC5) contributes to cell migration and invasion and is directly regulated by tumor-suppressive microRNA-133a in head and neck squamous cell carcinoma. Int J Oncol. 2012; 40: 1770-8.

23. Sarmiento C, Wang W, Dovas A, et al. WASP family members and formin proteins coordinate regulation of cell protrusions in carcinoma cells. J Cell Biol. 2008; 180: 1245-60.

24. Arimura N, Menager C, Fukata Y, et al. Role of CRMP-2 in Neuronal Polarity. J Neurobiol. 2004; 58: 34-47. 